\title{
ARTICLE Intrinsic functional connectivity correlates of person-level risk for bipolar disorder in offspring of affected parents
}

Danella M. Hafeman (iD ${ }^{1}$, Henry W. Chase ${ }^{1}$, Kelly Monk ${ }^{1}$, Lisa Bonar ${ }^{1}$, Mary Beth Hickey ${ }^{1}$, Alicia McCaffrey ${ }^{1}$, Simona Graur ${ }^{1}$, Anna Manelis ${ }^{1}$, Cecile D. Ladouceur ${ }^{1}$, John Merranko ${ }^{1}$, David A. Axelson ${ }^{2}$, Benjamin I. Goldstein ${ }^{3}$, Tina R. Goldstein ${ }^{1}$, Boris Birmaher ${ }^{1}$ and Mary L. Phillips ${ }^{1}$

Offspring of parents with bipolar disorder (OBP) are at increased risk to develop bipolar disorder (BD). Alterations in resting-state functional connectivity ( $\mathrm{rSFC}$ ) have been identified in OBP; however, replication has been limited and correlation with person-level risk is unknown. A recent study found reduced rsFC between left inferior frontal gyrus (IFG) and clusters in the left insula (LINS), lentiform nucleus (LENT), and midcingulate cortex (MCING) in OBP (Roberts et al. 2017); here, we aim to extend these findings to atrisk youth. We scanned a subset of the Pittsburgh Bipolar Offspring Study, a longitudinal study of OBP and community controls. Twenty-four OBP, 20 offspring of control parents with non-bipolar psychopathology (OCP), and 27 healthy controls (HC) had acceptable rsFC data. After preprocessing steps, we assessed group differences in seed-based rsFC between the IFG and target clusters (LINS, LENT, MCING) using multivariate regression. Next, we tested whether rsFC correlated with person-level risk score and with other dimensional measures. We did not find group differences in rsFC between IFG and target regions. Within OBP, risk score negatively correlated with IFG-LINS rsFC $(p=0.002)$. Across groups, mood lability correlated negatively with rsFC between IFG and target regions $(p=0.0002)$, due to negative correlation with IFG-LINS $(p=0.0003)$ and IFG-MCING $(p=0.001)$ rsFC. While grouplevel differences were not replicated, IFG-LINS rsFC was negatively correlated with a person-level risk score in OBP and with mood lability (a predictor of BD) across the sample. Thus, IFG-LINS rsFC might constitute a risk marker, within OBP, for the development of BD.

Neuropsychopharmacology (2019) 44:629-634; https://doi.org/10.1038/s41386-018-0264-9

\section{INTRODUCTION}

Offspring of parents with bipolar disorder (OBP) are at increased risk to develop the disorder themselves, but still only a minority will eventually meet full criteria for bipolar disorder (BD). In the Pittsburgh Bipolar Offspring Study (BIOS), the cumulative incidence of bipolar spectrum disorder (BPSD) was $25 \%$ by early adulthood, while the cumulative incidence of full-threshold bipolar-I or -II was $\sim 13 \%$ [1]. Recent work has focused on identifying predictors of risk in these youth, which include mood lability, depression, anxiety, and (closer to onset) subthreshold manic symptoms, especially in the context of a parent with earlyonset mood disorder [2-4]. We have combined extant risk factors from the literature into a risk calculator that is able to distinguish youth who will develop BPSD within the next five years versus those who will not [5]. Thus each at-risk youth has a "risk score" (between 0 and 1) that quantifies person-level risk for progression. An important next step is to assess how individual risk score may correlate with neurobiological changes, to begin to identify markers of risk and resilience. This also sets the stage for later assessing whether brain markers might improve individual prediction.

A growing body of literature points to the inferior frontal gyrus (IFG) and closely related ventrolateral prefrontal cortex (VLPFC) as key elements of neural circuitry underlying the development of $\mathrm{BD}$
[6]. These regions have been implicated in subjective reward value encoding, cognitive control, and emotion regulation [7-9], all behavioral processes that have been shown to be abnormal in individuals with $\mathrm{BD}$ and their first-degree relatives. The majority of extant literature has focused on differences in activation and taskrelated functional connectivity between at-risk offspring and controls, using reward [10], emotion regulation [11], emotional nback task [12], and affective go/no-go tasks [13]. Many of these studies have found differences in activation and/or task-related functional connectivity between individuals at familial risk for BD and healthy controls within the IFG and VLPFC.

Abnormalities in resting-state (task-free, intrinsic) functional connectivity ( $\mathrm{rSFC}$ ) have also been found in individuals at familial risk for BD [14-19], and again, the IFG and VLPFC emerge as key regions that distinguish at-risk and healthy controls [14, 15] (Table S1). In healthy, young offspring of a bipolar parent (vs. healthy controls), Singh and co-authors [14] found less VLPFCcaudate rsFC and more rsFC within the VLPFC, although the latter was associated with higher functioning [15]. In a sample of young adult at-risk individuals with BD, some of whom already had psychopathology (vs. community controls), Roberts and colleagues [14] found that the left IFG was less connected to a network of regions: left insula (LINS), midcingulate cortex (MCING), and lentiform nucleus (LENT) [14]. They also found a similar, though

\footnotetext{
${ }^{1}$ University of Pittsburgh, 38110 Hara St, Pittsburgh, PA 15213, USA; ${ }^{2}$ Nationwide Children's Hospital and The Ohio State University, Columbus, OH, USA and ${ }^{3}$ Sunnybrook Health Sciences Centre, University of Toronto, Toronto, Canada

Correspondence: Danella M. Hafeman (hafemand@upmc.edu)
}

Received: 28 June 2018 Revised: 5 October 2018 Accepted: 28 October 2018

Published online: 8 November 2018 
more pronounced, pattern of decreased rsFC between the left IFG and a broader network of cortical regions (including bilateral insula) in young adults with BD [14]. When interpreting previous findings, it is important to view OBP as a heterogeneous population that may differ according to "stage" of risk, e.g., whether OBP are healthy (e.g., Stage 0) and perhaps resilient, or already have possible prodromal symptoms of BD (e.g., Stage 1a/ 1b) [20]. Such clinical heterogeneity may explain differences across studies, and also motivates the assessment of person-level risk differences (and correlated neural markers) in at-risk samples.

Here, we aim to replicate and extend findings from the Roberts et al. [14] study, which found reduced rsFC between left IFG and three target regions (LINS, MCING, and LENT) in OBP vs. HC. Reproducibility is a current concern across scientific fields that has especially been problematic in neuroimaging, given fairly small sample sizes, diverse protocols, and variable processing streams [21]. Thus, we first test whether findings replicate in a younger sample (7-17 years old vs. 16-30 years old), also assessing differences in developmental trajectories of IFG-target region rsFC (age ${ }^{*}$ group interactions). BIOS is not a perfect replication sample, given important differences between the samples (e.g., depression rates, antidepressant use; see Table S1); however, in both samples, a substantial proportion of at-risk participants had psychopathology and were on psychotropic medication. In addition, the Roberts et al. [14]. study had a large sample ( $n>70$ for at-risk and healthy controls), well-described and rigorous methodology, and robust findings.

Next, we extend these findings to assess whether rsFC between IFG and target regions differs according to risk score and other dimensional predictors of BPSD. Given clinical heterogeneity in $\mathrm{OBP}$, testing the relationship between brain markers and a quantitative measure of person-level risk for BD (i.e., risk score) may further strengthen inference regarding neural markers of risk.

\section{METHODS}

Sample

Participants were a subset of BIOS, an ongoing longitudinal study consisting of offspring of parents with BD-I or -II and offspring of community controls [22]. Youth 7-17 years old at time of scan who did not have BPSD were invited to participate. Exclusion criteria for all participants were: systemic medical illness, neurological disorders, head trauma, alcohol or illicit substance use, standard exclusion criteria for magnetic resonance imaging (MRI) research (metal in the head or body, claustrophobia, etc.), and intelligence quotient (IQ) $<70$ (using the Weschler Abbreviated Scale of Intelligence); siblings were also excluded. Eighty youth were scanned from this cohort: this included 32 offspring of bipolar parents (OBP), 31 offspring of parents with other psychopathology (offspring of control parents; OCP), and 17 healthy controls $(\mathrm{HC})$. We combined healthy controls from another study, the Longitudinal Assessment of Manic Symptoms [23] ( $n=$ 20), which had an identical neuroimaging protocol. Youth who moved $>3 \mathrm{~mm}$ were excluded from additional analyses. In addition, youth with $<4.5 \mathrm{~min}$ of "good" data (following "scrubbing" points with framewise displacement $>0.5 \mathrm{~mm}$; see procedures below) were excluded. This yielded a sample size of 24 OBP, 20 OCP, and $27 \mathrm{HC}$ (Table 1). Included youth were older than those who were excluded but did not differ according to group or other demographic factors (Table S2).

\section{Procedures}

BIOS study procedures have been described previously [11, 22]. Informed consent from the parents and assent from the children were obtained for both the overarching longitudinal study and the neuroimaging study. As part of the larger study, participating parents were assessed using the Structured Clinical Interview for DSM-IV. At each study visit (approximately every two years),

\begin{tabular}{|c|c|c|c|c|c|}
\hline & $\begin{array}{l}\text { OBP } \\
(n=24)\end{array}$ & $\begin{array}{l}\text { OCP } \\
(n=20)\end{array}$ & $\begin{array}{l}\mathrm{HC} \\
(n=27)\end{array}$ & Stat & $p$ \\
\hline Female $n(\%)$ & $14(58 \%)$ & $12(60 \%)$ & $13(48 \%)$ & $X^{2}=0.82$ & 0.66 \\
\hline Age in years (S.D.) & $14.3(2.3)$ & $13.8(2.3)$ & $14.2(1.9)$ & $F=0.28$ & 0.76 \\
\hline Right Handed $n(\%)$ & $21(88 \%)$ & $18(90 \%)$ & $24(89 \%)$ & a & 0.99 \\
\hline SES (S.D.) & $2.96(1.20)$ & $3.50(1.64)$ & $\begin{array}{l}3.74 \\
(1.20)\end{array}$ & $\mathrm{F}=2.25$ & 0.11 \\
\hline IQ (S.D.) & $99.1(15.7)$ & $\begin{array}{l}100.5 \\
(13.4)\end{array}$ & $\begin{array}{l}104.7 \\
(13.5)\end{array}$ & $F=1.04$ & 0.36 \\
\hline $\begin{array}{l}\text { Psychiatric } \\
\text { diagnosis } n \text { (\%) }\end{array}$ & $9(38 \%)$ & $10(50 \%)$ & - & $X^{2}=0.69$ & 0.40 \\
\hline $\begin{array}{l}\text { Depression } n \\
\text { (\%) }\end{array}$ & $1(4 \%)$ & $2(10 \%)$ & - & & \\
\hline Anxiety $n(\%)$ & $3(12 \%)$ & $4(20 \%)$ & - & & \\
\hline OCD $n(\%)$ & $0(0 \%)$ & $2(10 \%)$ & - & & \\
\hline ADHD $n(\%)$ & $4(17 \%)$ & $4(20 \%)$ & - & & \\
\hline DBD & $1(4 \%)$ & $2(10 \%)$ & - & & \\
\hline \begin{tabular}{|l} 
Psychotropic \\
medication $n(\%)$
\end{tabular} & $2(8 \%)$ & $5(25 \%)$ & - & a & 0.22 \\
\hline $\begin{array}{l}\text { Antidepressants } \\
n(\%)\end{array}$ & $0(0 \%)$ & $2(10 \%)$ & - & & \\
\hline Stimulants $n(\%)$ & $1(4 \%)$ & $3(15 \%)$ & - & & \\
\hline $\begin{array}{l}\text { Non-stimulants } \\
n(\%)\end{array}$ & $1(4 \%)$ & $1(5 \%)$ & - & & \\
\hline
\end{tabular}

parents and their offspring were interviewed using the Schedule for Affective Disorders and Schizophrenia for School Age ChildrenPresent and Lifetime Version (K-SADS-P/L) for non-mood disorders and the K-SADS Mania Rating Scale (KMRS) and the depression items from the KSADS-Present Version (KDRS), which assess subthreshold and threshold mood symptoms [24, 25]. Self- and parent-reports of depressive symptoms over the past two weeks (Mood and Feelings Questionnaire; MFQ) [26], anxiety over the past three months (Screen for Child Anxiety Related Disorders; SCARED) [27], and mood lability (Child Affective Lability Scale; CALS) [28] were also collected. Based on assessments administered at study visit, risk score was calculated as previously described [5], based on predictors from a meta-analysis by Van Meter et al. [29] (e.g., subthreshold manic symptoms, subthreshold depressive symptoms, mood lability, and anxiety); we also included age and parental age of onset, based on previous literature [30, 31].

As part of a 90-min neuroimaging session, youth underwent a 6min resting-state protocol with eyes open on a Siemens Trio scanner $(T R=2000 \mathrm{~ms}, T E=28 \mathrm{~ms})$. On day of scan, $M F Q$, SCARED, and CALS were collected (self- and parent-reports); information was also obtained about handedness [32] and socioeconomic status [33]. All participants had a study visit within 18 months of scan, with most study visits being within the previous 6 months. All study procedures were approved by the University of Pittsburgh Institutional Review Board.

\section{Data analysis}

Data were preprocessed using DPABI, an SPM-based platform that implements a flexible pipeline with state-of-the-art motion correction [34]. The first four time points were removed to allow for image stabilization, followed by slice timing correction and realignment. Co-registration was implemented using the "new segment" protocol and DARTEL. Next, regression was performed to regress out artifact; we used the Friston 24-parameter model to correct for motion $[35,36]$, as well as using CompCor to regress 
A

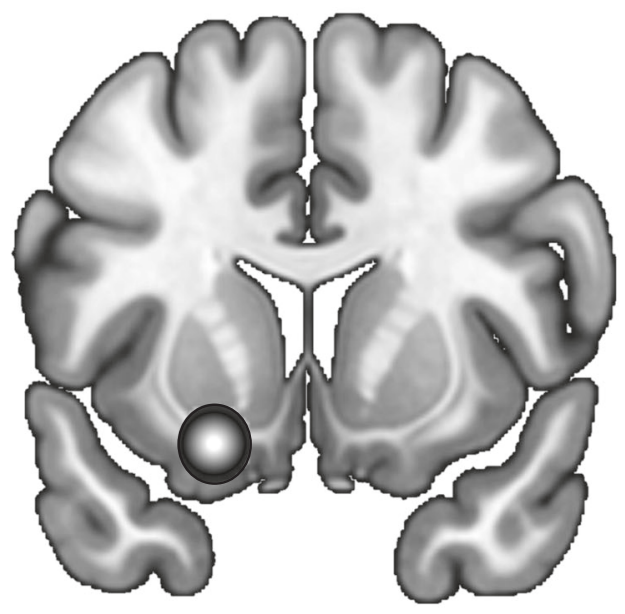

B

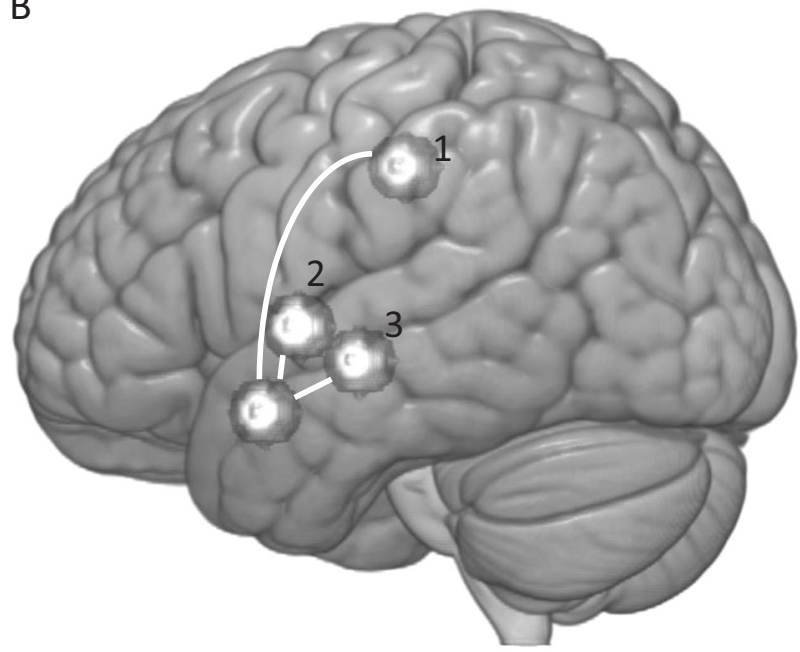

Fig. 1 Left inferior frontal gyrus (LIFG) seed (a) and target regions (b). Target regions are [1] midcingulate (MCING), [2] left insula (LINS), and [3] left lentiform nucleus (LENT)

out white matter and CSF signal [37], and scrubbing regressors to effectively remove any time point with sudden movement (framewise displacement [38] $>0.5 \mathrm{~mm}$ ) as well as the time point previous. Due to the debate on global signal regression, we ran analyses with and without this variable [39]; findings did not differ, and here we report the results corrected for global signal. Images were normalized by DARTEL, smoothed using a $6 \mathrm{~mm}$ smoothing kernel, and filtered $(0.01-0.08 \mathrm{~Hz})$. Because the Roberts et al. analysis did not apply a filter, we also reran analyses without a filter; findings remained unchanged.

We extracted correlations between timeseries in seed and target spheres, centered on coordinates reported in Roberts et al. $[13,14]$ and a radius of $8 \mathrm{~mm}$. We used a LIFG seed (-21 $11-17)$; target regions were LINS $(-35-13)$, MCING $(-7-1843)$, and LENT $(-25-12-5)$ (Fig. 1). We chose an $8 \mathrm{~mm}$ radius because this was most consistent with the number of voxels reported by Roberts et al. in the LIFG seed.

In SAS 9.4, we implemented multivariate regression using proc $\mathrm{g} / \mathrm{m}$ to test whether seed-target region rsFC differed according to group. For the primary analysis, dependent variables were LIFGLINS correlation, LIFG-MCING correlation, and LIFG-LENT correlation. Effects of age and age $\times$ group interactions were also tested. Next, we assessed, in the OBP group, whether IFG-target rsFC was correlated with risk score. Finally, we tested, across the whole sample, whether rsFC correlated with depression, anxiety, and mood lability (average report of parent and child: MFQpc, SCAREDPc, and CALSpc) at time of scan, after adjusting for group. We initially tested these dimensions in separate models, then together to test for which dimensions were independently associated with IFG-target rsFC. All analyses were adjusted for age, gender, and mean framewise displacement (FD-Power [38]); all full-sample dimensional analyses were also adjusted for group. We also subsequently controlled analyses for psychiatric diagnosis and medication, to assess whether observed effects were attributable to these variables. To ensure that dimensional findings were not driven by outliers, we repeated analyses after removing values $>1.96$ standard deviations above the scale mean. We report Wilks' Lambda for the multivariate regressions, which simultaneously models the three connectivity coefficients of interest (and thus adjusts for multiple comparisons); for models with Wilks' Lambda $p<0.05$, we report individual target regions. To account for differing scan lengths (due to "scrubbing"), we also conducted a weighted regression in SAS, weighting according to scan duration.

We also conducted exploratory whole-brain analyses in SPM12 (cluster-forming threshold $p=0.001, k \geq 25$ voxels) to test for group differences, correlations with risk score, and correlations with other dimensions. Cluster-wise FWE-corrected $p$-values given by SPM12 are reported, unless otherwise noted.

\section{RESULTS}

Between-group analyses

The rsFC between IFG and target regions did not significantly differ across groups $(\Lambda=0.86, \mathrm{~F}=1.60, p=0.15)$; there were no significant pairwise differences between OBP and other groups. There was no main effect of age and age $\times$ group interactions were not significant. Adjustment for psychiatric diagnosis and medication variables did not alter findings; weighting according to uncensored scan time also did not change results (Table S3).

Risk score analysis

Within OBP, rsFC between LIFG and target regions were significantly correlated with risk score $(p=0.02$, Table 2$)$. This was driven entirely by a negative correlation between IFG-LINS and risk score $(r=-0.64, p=0.002$; Fig. 2a). This correlation remained significant after adjusting for psychiatric diagnosis (yes/ no) and medication (yes/no) at time of scan; weighting analyses by scan time did not change results (Table S3).

Risk score is a composite of clinical scales (e.g., CALSpc and SCAREDpc), as well as the parental age of mood disorder onset (where early age onset in parent is a predictor of offspring disorder). To probe which components of risk score might be driving the LIFG-LINS relationship, we ran an additional model including relevant components of risk score (parent age of onset, CALSpc, and SCAREDpc) and standard covariates. Older parent age of onset (a protective factor) was positively correlated with LIFG-LINS rsFC $(r=0.46, p=0.046$; eFigure 1$)$; CALSpc and SCAREDPC were no longer significant in this model.

Dimensions analysis

Across the sample (and adjusting for family history), rsFC between LIFG and target regions was negatively correlated with mood lability (CALSpc) and, to a lesser extent, anxiety (SCAREDpc) (Table 2). LIFG-LINS rsFC was negatively correlated with CALSpC $(r=-0.43, p=0.0003)$, even after removing outliers; LIFG-MCING rsFC was also negatively correlated with CALSpc $(r=-0.40, p=$ 0.001 ; Fig. 2c), though this relationship was no longer significant when outliers were removed. Both LIFG-LINS and LIFG-MCING rsFC was also negatively correlated with SCAREDpc; however, these relationships were less robust to multiple comparisons (particularly after the removal of outliers) (Table 2), and diminished considerably with adjustment for diagnosis (Table S3). MFQpc was 
Table 2. Association between each dimension and multivariate outcome (LIFG-LINS, LIFG-LENT, and LIFG-MCING rsFC)

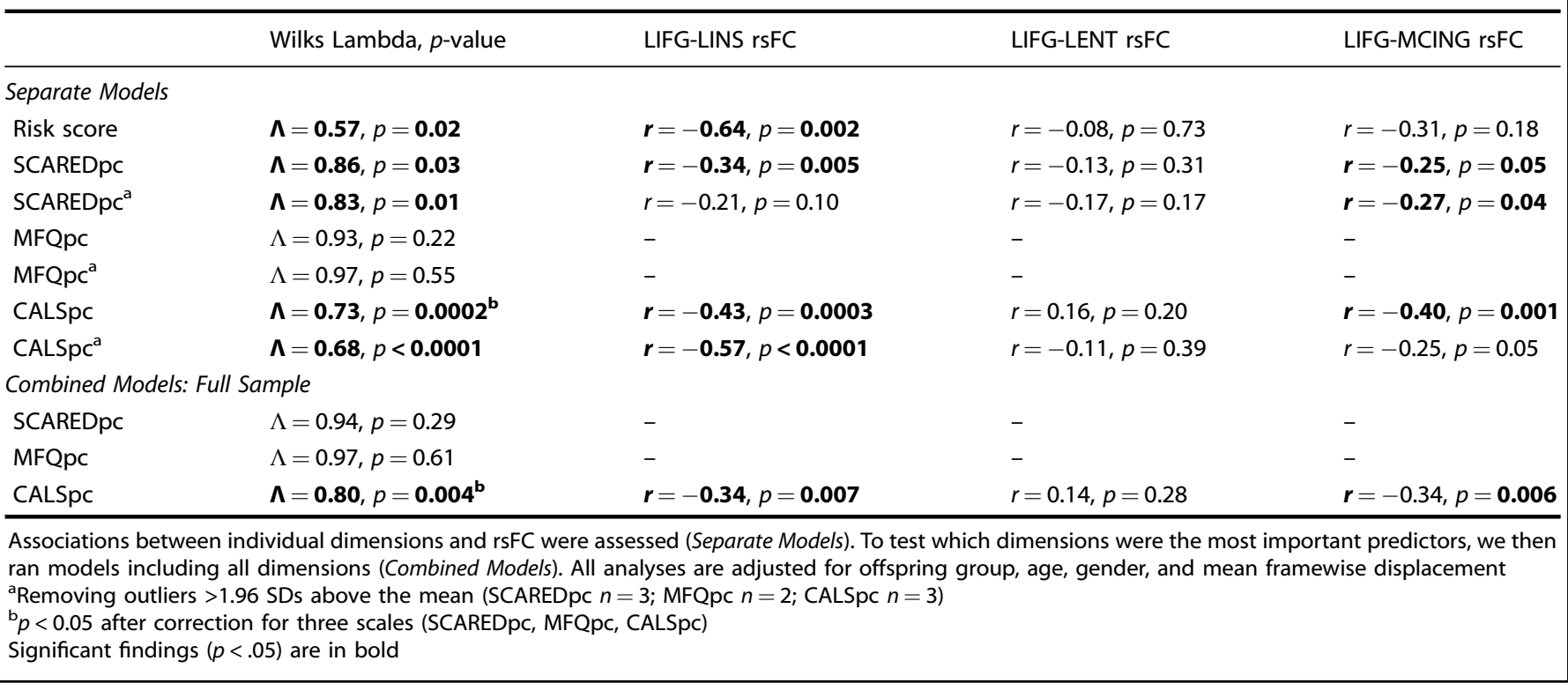

not correlated with LIFG rsFC. Adjustment for psychiatric medication did not alter these findings, nor did weighting by scan time (Table S3); dimension $\times$ group interactions were not significant, indicating similar correlation regardless of risk group.

In a single model including all dimensional predictors (CALSpc, SCAREDpc, and MFQpc), CALSpc remained significantly correlated with rsFC between LIFG and target regions $(p=0.004)$, due to significant correlations with both LIFG-MCING and LIFG-LINS rsFC; SCAREDpc was no longer significant (Table 2).

Whole-brain analyses: LIFG seed

There were no significant group differences in rsFC between LIFG and whole brain. We also did not observe significant correlations between LIFG rsFC and risk score at the whole-brain level. CALSpc was positively correlated with LIFG-superior frontal gyrus rsFC (2 clusters: $k=48$, FWE-corrected $p=0.02 ; k=44$, FWE-corrected $p=0.03)$ and negatively correlated with IFG-left postcentral gyrus rsFC ( $k=52$, FWE-corrected $p=0.02$ ) (Table S4; Figure S2). At the whole-brain level, CALSpc showed negative correlations with LIFGLINS rsFC, though this did not survive FWE correction $(k=32$, FWE-corrected $p=0.11)$. SCAREDpc was positively correlated with IFG-right superior frontal gyrus $\mathrm{rsFC}(k=68$, FWE-corrected $p=$ 0.004). We did not observe negative correlations between SCAREDpc and LIFG-target region rsFC. There were no (FWEcorrected) significant correlations between MFQpc and LIFG rsFC (Table S4).

\section{DISCUSSION}

We aimed to replicate and extend findings from a recent study that showed reduced rsFC between LIFG and three target regions (LINS, MCING, and LENT) in individuals at familial risk for BD [14]. While we did not find group differences in rsFC between these regions, as previously reported, we did find compelling correlations between LIFG-target region rsFC and both risk score (in the OBP) and mood lability (across the sample). Specifically, we found that LIFG-LINS rsFC was negatively correlated with risk score, and that LIFG-LINS (and, to a lesser extent, LIFG-MCING) rsFC was negatively correlated with mood lability. We did find that anxiety was also inversely correlated with LIFG-LINS and LIFG-MCING rsFC, but these findings were not significant after accounting for mood lability or psychiatric diagnosis. Given the lack of separation according to family history, one important possibility is that the LIFG-LINS is related to psychopathology in general, and not specific risk for BD. However, we found young parental age of mood disorder onset (an important, symptom-independent predictor of BD in at-risk offspring) to also predict less LIFG-LINS rsFC. This provides further evidence that LIFG-LINS rsFC may be correlated with BD risk in OBP, above and beyond symptom counts. Across group (and adjusting for family history status), LIFG-LINS rsFC was also correlated with mood lability, a key dimensional predictor of BD risk. With the Roberts et al. [14] findings, we find further support for the hypothesis that LIFG rsFC is important for risk, at least partially through effects on mood lability. However, findings do need to be interpreted with caution, give the absence of hypothesized group (family history) differences.

The IFG seed utilized in this paper is a medial, ventral, and posterior region that includes parts of Brodmann Area (BA47), anterior insula, subcallosal gyrus, and orbitofrontal cortex. This seed was initially chosen by Roberts et al. [14] based on their previous finding that individuals at familial risk for BD showed reduced IFG recruitment when inhibiting response to fearful faces [13]. This region has also been implicated in delay discounting and is activated in trials in which the longer term (larger) reward is chosen over the short-term (smaller) reward [40], and is important for updating internal representations of stimulus-outcome relationships in the context of reward [41]. Both in response to reward and fearful faces, LIFG activation and functional connectivity may be key to regulating choice-related impulsivity, particularly in the face of emotional stimuli. In particular, we find that reduced LIFG rsFC to brain areas key for regulatory [42] (MCING) and interoceptive [43] (LINS) processes is associated with mood lability. One interpretation is that the dysconnectivity of the LIFG from regulatory/interoceptive networks leads to elevated emotion-related impulsivity, which results in emotion dysregulation and mood lability. This dysconnectivity, particularly from the LINS, also appears to increase risk of BD in OBP.

Thus, our findings are consistent with the possibility that rsFC of this IFG seed (particularly with LINS and MCING) is a neural marker for increased mood lability due to difficulty inhibiting response to emotional stimuli. This has broader applicability in child psychiatry, as mood lability is a fairly common presenting problem 

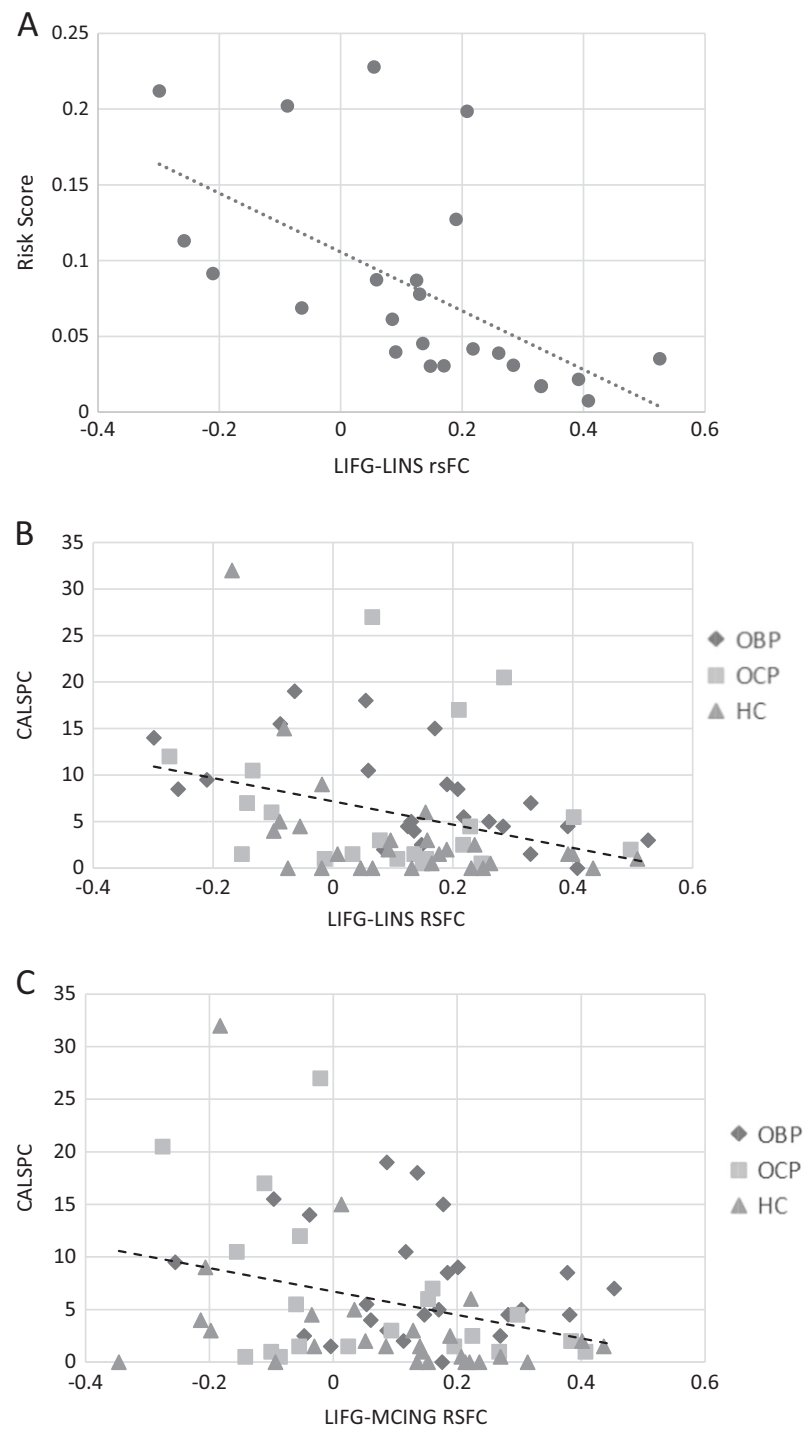

Fig. 2 Correlations between LIFG and symptom dimensions. a Correlation between LIFG-LINS rsFC and risk score in OBP $(n=24$; $r=-0.64, p=0.002$ ). b Correlation between LIFG-LINS rsFC and CALSpc in the full sample $(n=71 ; r=-0.43, p=0.0003)$. c Correlation between LIFG-MCING rsFC and CALSpc in the full sample $(n=71 ; r=-0.40, p=0.001)$. LIFG left inferior frontal gyrus, LINS left insula, CALSpc Child Affective Lability Scale, OBP offspring of bipolar parents, OCP offspring of control parents, $\mathrm{HC}$ healthy controls

that causes problems with psychosocial functioning and increases risk for the development of both internalizing and externalizing disorders [44]. Mood lability is also higher in OBP and predictive of $\mathrm{BD}$ in youth at familial risk $[2,45]$. If these findings are replicated, future work might use IFG rsFC as a neural target of treatments aiming to decrease emotion-related impulsivity and mood lability.

Interestingly, we were not able to replicate group-level findings from the Roberts et al. analysis. One explanation for this lack of replication is that the Roberts sample was further along the bipolar risk continuum than our sample, in part related to age differences. Our participants were 7-17 years old with low rates of anxiety and depression; in contrast, the participants in the previous study were 16-30 years old with (as expected) higher rates of anxiety and depression. It is possible that reduced IFGtarget region rsFC becomes more ubiquitous and thus a marker of family risk in late adolescence, whereas in childhood and early adolescence, this marker instead distinguishes those who are developmentally at the highest risk of conversion (e.g., with an early-onset parent). Other explanations for this discrepancy are also possible. First, our sample was small relative to the previous study, and the absence of group-level differences might be related to limited power to replicate these findings. Post hoc power calculations indicate that, given our sample size, our power to detect the effect size observed in Roberts et al. [14] was 70\% for LIFG-LINS (see Supplement). Second, we did not fully replicate the Roberts et al. methods, but rather used published coordinates and an independent pipeline to assess group- and risk-related differences. It is possible that the lack of full replication is due to methodological differences. Despite this different sample, agegroup, and analytic method, our findings do, from a broader perspective, support the hypothesis that IFG rsFC, particularly with the LINS, is a potential risk marker in OBP.

The current findings must be interpreted considering the following limitations. First, the sample size of youth in the OBP and OCP groups was rather small (24 and 20 participants, respectively) which is why we chose a direct replication rather than a more exploratory analysis. Second, we had to exclude many participants (29 of 100 scanned) due to excessive movement. This might limit the generalizability of our findings, although youth who were excluded vs. included did not differ according to group (Table S2). Also, given the noise that inclusion of these participants would add to our findings, it is preferable to exclude them. Third, with rsFC, there is the possibility that micro-movements and other artifact can cause systematic noise. We found that groups did not differ according to mean framewise displacement, a measure of sudden movements, thus decreasing this possibility; similarly, framewise displacement did not correlate with CALSpc or risk score. Fourth, a scan length of $6 \mathrm{~min}$, while standard in the field, is somewhat short. However, previous work indicates that networks stabilize within $\sim 5$ min [46]; in addition, when we reran analyses down-weighting shorter scans, results were unchanged.

In conclusion, we find that LIFG rsFC with target regions, previously found to be reduced in individuals at familial risk for $\mathrm{BD}$, is negatively correlated with risk score (in OBP) and mood lability (across the sample). While we did not replicate LIFG rsFC as a marker of familial risk in this younger sample with less anxiety/ depression, we did find evidence that LIFG-LINS rsFC is an important marker of person-level risk within offspring of parents with $\mathrm{BD}$, as well as possibly a broader marker of emotion-related impulsivity. If replicated, LIFG-LINS rsFC might represent a potential marker of mood lability, a marker of individual risk in those with a family history of $\mathrm{BD}$, and a potential neural target for early intervention.

\section{FUNDING AND DISCLOSURE}

This work was supported by grants from the National Institute of Health 2 R01 MH060952-12S1 to Birmaher, Axelson, Phillips (MPIs) and R01 MH073953 to Birmaher and Phillips (MPIs). Dr. Hafeman receives funding from the NIMH and Klingenstein Third Generation Foundation. Dr. Axelson receives royalties from UpToDate. Dr. T. Goldstein receives funding from NIMH, The American Foundation for Suicide Prevention, The Brain and Behavior Foundation and the University of Pittsburgh Clinical and Translational Science Institute, and royalties from Guilford Press. Dr. Birmaher receives funding from the $\mathrm{NIMH}$; he receives royalties from Random House, Lippincott Williams \& Wilkins, and UpToDate. Dr. Phillips and Dr. Chase receive funding from NIMH.

\section{ACKNOWLEDGEMENTS}

We would like to acknowledge all the participants and their families for their contribution to this study. 


\section{ADDITIONAL INFORMATION}

Supplementary Information accompanies this paper at (https://doi.org/10.1038/ s41386-018-0264-9).

Competing interests: The authors declare no competing interests.

Publisher's note: Springer Nature remains neutral with regard to jurisdictional claims in published maps and institutional affiliations.

\section{REFERENCES}

1. Axelson D, Goldstein B, Goldstein T, Monk K, Yu H, Hickey MB, et al. Diagnostic precursors to bipolar disorder in offspring of parents with bipolar disorder: a longitudinal study. Am J Psychiatry. 2015;172:638-46.

2. Hafeman DM, Merranko J, Axelson D, Goldstein BI, Goldstein T, Monk K, et al. Toward the definition of a bipolar prodrome: dimensional predictors of bipolar spectrum disorders in at-risk youths. Am J Psychiatry. 2016;173:695-704.

3. Duffy A, Alda M, Hajek T, Sherry SB, Grof P. Early stages in the development of bipolar disorder. J Affect Disord. 2010;121:127-35.

4. Mesman E, Nolen WA, Keijsers L, Hillegers MHJ. Baseline dimensional psychopathology and future mood disorder onset: findings from the Dutch Bipolar Offspring Study. Acta Psychiatr Scand. 2017;136:201-9.

5. Hafeman DM, Merranko J, Goldstein TR, Axelson D, Goldstein BI, Monk K, et al. Assessment of a person-level risk calculator to predict new-onset bipolar spectrum disorder in youth at familial risk. JAMA Psychiatry. 2017:74:841-7.

6. Phillips ML, Swartz HA. A critical appraisal of neuroimaging studies of bipolar disorder: toward a new conceptualization of underlying neural circuitry and a road map for future research. Am J Psychiatry. 2014;171:829-43.

7. Rudebeck PH, Saunders RC, Lundgren DA, Murray EA. Specialized representations of value in the orbital and ventrolateral prefrontal cortex: desirability versus availability of outcomes. Neuron. 2017;95:1208-20.e1205.

8. Levy BJ, Wagner AD. Cognitive control and right ventrolateral prefrontal cortex: reflexive reorienting, motor inhibition, and action updating. Ann N Y Acad Sci. 2011;1224:40-62.

9. Wager TD, Davidson ML, Hughes BL, Lindquist MA, Ochsner KN. Prefrontalsubcortical pathways mediating successful emotion regulation. Neuron. 2008;59:1037-50.

10. Manelis A, Ladouceur CD, Graur S, Monk K, Bonar LK, Hickey MB, et al. Altered functioning of reward circuitry in youth offspring of parents with bipolar disorder. Psychol Med. 2015;46:197-208.

11. Manelis A, Ladouceur CD, Graur S, Monk K, Bonar LK, Hickey MB, et al. Altered amygdala-prefrontal response to facial emotion in offspring of parents with bipolar disorder. Brain. 2015

12. Ladouceur CD, Diwadkar VA, White R, Bass J, Birmaher B, Axelson DA, et al. Fronto-limbic function in unaffected offspring at familial risk for bipolar disorder during an emotional working memory paradigm. Dev Cogn Neurosci. 2013;5:185-96.

13. Roberts G, Green MJ, Breakspear M, McCormack C, Frankland A, Wright A, et al. Reduced inferior frontal gyrus activation during response inhibition to emotional stimuli in youth at high risk of bipolar disorder. Biol Psychiatry. 2013;74:55-61.

14. Roberts G, Lord A, Frankland A, Wright A, Lau P, Levy F, et al. Functional dysconnection of the inferior frontal gyrus in young people with bipolar disorder or at genetic high risk. Biol Psychiatry. 2017

15. Singh MK, Chang KD, Kelley RG, Saggar M, Reiss AL, Gotlib IH. Early signs of anomalous neural functional connectivity in healthy offspring of parents with bipolar disorder. Bipolar Disord. 2014;16:678-89.

16. Doucet GE, Bassett DS, Yao N, Glahn DC, Frangou S. The role of intrinsic brain functional connectivity in vulnerability and resilience to bipolar disorder. Am J Psychiatry. 2017;174:1214-22

17. Ford KA, Theberge J, Neufeld RJ, Williamson PC, Osuch EA. Correlation of brain default mode network activation with bipolarity index in youth with mood disorders. J Affect Disord. 2013;150:1174-8.

18. Meda SA, Gill A, Stevens MC, Lorenzoni RP, Glahn DC, Calhoun VD, et al. Differences in resting-state functional magnetic resonance imaging functional network connectivity between schizophrenia and psychotic bipolar probands and their unaffected first-degree relatives. Biol Psychiatry. 2012;71:881-9.

19. Lui S, Yao L, Xiao Y, Keedy SK, Reilly JL, Keefe RS, et al. Resting-state brain function in schizophrenia and psychotic bipolar probands and their first-degree relatives. Psychol Med. 2015;45:97-108.

20. Berk M, Post R, Ratheesh A, Gliddon E, Singh A, Vieta E, et al. Staging in bipolar disorder: from theoretical framework to clinical utility. World Psychiatry. 2017;16:236-44.
21. Poldrack RA, Baker Cl, Durnez J, Gorgolewski KJ, Matthews PM, Munafò MR, et al. Scanning the horizon: towards transparent and reproducible neuroimaging research. Nat Rev Neurosci. 2017;18:115.

22. Birmaher B, Axelson D, Monk K, Kalas C, Goldstein B, Hickey MB, et al. Lifetime psychiatric disorders in school-aged offspring of parents with bipolar disorder: the Pittsburgh Bipolar Offspring study. Arch Gen Psychiatry. 2009;66:287-96.

23. Horwitz SM, Demeter CA, Pagano ME, Youngstrom EA, Fristad MA, Arnold LE, et al. Longitudinal Assessment of Manic Symptoms (LAMS) study: background, design, and initial screening results. J Clin Psychiatry. 2010;71:1511-7.

24. Axelson D, Birmaher BJ, Brent D, Wassick S, Hoover C, Bridge J, et al. A preliminary study of the Kiddie Schedule for Affective Disorders and Schizophrenia for School-Age Children mania rating scale for children and adolescents. J Child Adolesc Psychopharmacol. 2003;13:463-70.

25. Kaufman J, Birmaher B, Brent D, Rao U, Flynn C, Moreci P, et al. Schedule for Affective Disorders and Schizophrenia for School-Age Children-Present and Lifetime Version (K-SADS-PL): initial reliability and validity data. J Am Acad Child Adolesc Psychiatry. 1997;36:980-8.

26. Daviss WB, Birmaher B, Melhem NA, Axelson DA, Michaels SM, Brent DA. Criterion validity of the Mood and Feelings Questionnaire for depressive episodes in clinic and non-clinic subjects. J Child Psychol Psychiatry. 2006;47:927-34.

27. Birmaher B, Brent DA, Chiappetta L, Bridge J, Monga S, Baugher M. Psychometric properties of the Screen for Child Anxiety Related Emotional Disorders (SCARED): a replication study. J Am Acad Child Adolesc Psychiatry. 1999;38:1230-6.

28. Gerson AC, Gerring JP, Freund L, Joshi PT, Capozzoli J, Brady K, et al. The Children's Affective Lability Scale: a psychometric evaluation of reliability. Psychiatry Res. 1996;65:189-98.

29. Van Meter AR, Burke C, Youngstrom EA, Faedda GL, Correll CU. The bipolar prodrome: meta-analysis of symptom prevalence prior to initial or recurrent mood episodes. J Am Acad Child \& Adolesc Psychiatry. 2016;55:543-55.

30. Baldessarini RJ, Tondo L, Vazquez GH, Undurraga J, Bolzani L, Yildiz A, et al. Age at onset versus family history and clinical outcomes in 1,665 international bipolar-I disorder patients. World Psychiatry. 2012;11:40-6.

31. Lin P-I, Mclnnis MG, Potash JB, Willour V, MacKinnon DF, DePaulo JR, et al. Clinical correlates and familial aggregation of age at onset in bipolar disorder. Am J Psychiatry. 2006;163:240-6.

32. Oldfield RC. The assessment and analysis of handedness: The Edinburgh inventory. Neuropsychologia. 1971;9:97-113.

33. Hollingshead AB. Four-factor index of social status. New Haven, Connecticut: Yale University Department of Sociology; 1975.

34. Yan C-G, Wang X-D, Zuo X-N, Zang Y-F. DPABI: data processing \& analysis for (resting-state) brain imaging. Neuroinformatics. 2016;14:339-51.

35. Yuan $\mathrm{L}$, He $\mathrm{H}$, Zhang $\mathrm{H}$, Zhong J. Evaluating the influence of spatial resampling for motion correction in resting-state functional MRI. Front Neurosci. 2016; 10:591.

36. Friston KJ, Williams S, Howard R, Frackowiak RS, Turner R. Movement-related effects in fMRI time-series. Magn Reson Med. 1996;35:346-55.

37. Behzadi Y, Restom K, Liau J, Liu TT. A component based noise correction method (CompCor) for BOLD and perfusion based fMRI. Neuroimage. 2007;37:90-101.

38. Power JD, Barnes KA, Snyder AZ, Schlaggar BL, Petersen SE. Spurious but systematic correlations in functional connectivity MRI networks arise from subject motion. Neuroimage. 2012;59:2142-54.

39. Murphy K, Fox MD. Towards a consensus regarding global signal regression for resting state functional connectivity MRI. Neuroimage. 2017;154:169-73.

40. Smith BJ, Monterosso JR, Wakslak CJ, Bechara A, Read SJ. A meta-analytical review of brain activity associated with intertemporal decisions: evidence for an anterior-posterior tangibility axis. Neurosci Biobehav Rev. 2018;86:85-98.

41. Boorman ED, Rajendran VG, O'Reilly JX, Behrens TE. Two anatomically and computationally distinct learning signals predict changes to stimulus-outcome associations in hippocampus. Neuron. 2016;89:1343-54.

42. Tolomeo S, Christmas D, Jentzsch I, Johnston B, Sprengelmeyer R, Matthews K, et al. A causal role for the anterior mid-cingulate cortex in negative affect and cognitive control. Brain. 2016;139:1844-54.

43. Gu X, Hof PR, Friston KJ, Fan J. Anterior insular cortex and emotional awareness. J Comp Neurol. 2013;521:3371-88.

44. Stringaris A, Goodman R. Mood lability and psychopathology in youth. Psychol Med. 2009:39:1237-45.

45. Birmaher B, Goldstein Bl, Axelson DA, Monk K, Hickey MB, Fan J, et al. Mood lability among offspring of parents with bipolar disorder and community controls. Bipolar Disord. 2013;15:253-63.

46. Van Dijk KR, Hedden T, Venkataraman A, Evans KC, Lazar SW, Buckner RL. Intrinsic functional connectivity as a tool for human connectomics: theory, properties, and optimization. J Neurophysiol. 2010;103:297-321. 